\title{
INVARIANT MEANS FOR THE WOBBLING GROUP
}

\author{
KATE JUSCHENKO, MIKAEL DE LA SALLE
}

\begin{abstract}
Given a metric space $(X, d)$, the wobbling group of $X$ is the group of bijections $g: X \rightarrow X$ satisfying $\sup d(g(x), x)<\infty$. We study algebraic and analytic properties of $W(X)$ in relation with the metric space structure of $X$, such as amenability of the action of the lamplighter group $\bigoplus_{X} \mathbb{Z} / 2 \mathbb{Z} \rtimes W(X)$ on $\bigoplus_{X} \mathbb{Z} / 2 \mathbb{Z}$ and property $(\mathrm{T})$.
\end{abstract}

\section{INTRODUCTION}

In this paper we deal with amenable actions of discrete groups. In our setting an action of a group $G$ on a set $X$ is called amenable if there is an $G$-invariant mean on $X$. A linear map $\mu$ on $\ell_{\infty}(X)$ is a mean on $X$ if it is unital and $\|\mu\|=1$. A group $G$ is amenable if and only if its action on itself by left translation is amenable, in this case all actions of $G$ are amenable. Thus the question of determining whether an action is amenable is interesting in the case when $G$ is not (known to be) amenable.

Let $G$ be a discrete group acting transitively on a set $X$. The abelian group $\bigoplus_{X} \mathbb{Z} / 2 \mathbb{Z}$ carries an action of itself by translation, and an action of $G$ by permutation of the basis, which gives rise to an action of the semidirect product (also called permutational wreath product, or lamplighter group) $\bigoplus_{X} \mathbb{Z} / 2 \mathbb{Z} \rtimes G$. We will be interested in particular cases of the following general question :

Question 1.1. Is the action of $\bigoplus_{X} \mathbb{Z} / 2 \mathbb{Z} \rtimes G$ on $\bigoplus_{X} \mathbb{Z} / 2 \mathbb{Z}$ amenable?

An easy necessary condition for 1.1 is that the action of $G$ on $X$ is amenable. We observe that this is not sufficient, see Proposition 3.1.

In [10], Nekrashevych and the authors showed that Question 1.1 has a positive answer if the Schreier graph of the action of $G$ on $X$ is recurrent. However a characterization of the actions for which the answer is positive is still open.

In this note all metric spaces will be discrete. A metric space $X$ has bounded geometry if for every $R>0$, the balls of radius $R$ have bounded cardinality. We will mainly be interested in a special case of Question 1.1 when $(X, d)$ is a metric space with bounded geometry and $G$ is a group of bijections $g$ of $X$ with bounded displacement, i.e. with the property that $|g|_{w}<\infty$, where

$$
|g|_{w}:=\sup \{d(x, g(x)): x \in X\} .
$$

Following [5] (see also [3]) we will call the group of all such bijections of $X$ the wobbling group of $X$ and denote it by $W(X)$. In [1], [8, Remark 0.5. $\left.C_{1}^{\prime \prime}\right]$ and [5] the wobblings were introduced as tools to prove non-amenability results. In [9], they were used to prove amenability results (see below for details). When $X$ is a Cayley graph of a finitely generated group $\Gamma$ with word metric we will denote the wobbling group of $X$ shortly by $W(\Gamma)$. The group $W(\Gamma)$ does not

The work of M. de la Salle was partially supported by ANR grants OSQPI and NEUMANN. 
depend on a finite generating set of $\Gamma$ and it coincides with the group of piecewise translations of $\Gamma$. As a special case of Question 1.1 we can ask :

Question 1.2. Is the action of $\bigoplus_{X} \mathbb{Z} / 2 \mathbb{Z} \rtimes W(X)$ on $\bigoplus_{X} \mathbb{Z} / 2 \mathbb{Z}$ amenable?

The motivation for the question above is based on the recent result of the first named author and N. Monod, 9], where the authors show that the full topological group of Cantor minimal system is amenable, which was previously conjectured by Grigorchuk and Medynets in [7]. The combination of this result with the result of H. Matui, [12], produces the first examples of infinite simple finitely generated amenable groups. The technical core of [9] is to show that the Question 1.2 has a positive answer for the particular case $X=\mathbb{Z}$.

Our goal would be to give a necessary and sufficient condition on $(X, d)$ for Question 1.2 to have a positive answer. Theorem 1.4 summarizes our partial results in this direction.

Definition 1.3. Let $(X, d)$ be a metric space with bounded geometry and fix $x_{0} \in X$. $(X, d)$ is called transient if there is $R>0$ such that the random walk starting at $x_{0}$ and jumping from a point $x$ uniformly to $B(x, R)$ is transient. Otherwise it is called recurrent.

This notion does not depend on $x_{0}$, and when $(X, d)$ is a connected graph with graph distance this notion is equivalent to the transience of the usual random walk on this graph (Proposition 2.2).

Theorem 1.4. Let $(X, d)$ be a metric space with bounded geometry.

- If $(X, d)$ is recurrent, then the action of $\bigoplus_{X} \mathbb{Z} / 2 \mathbb{Z} \rtimes W(X)$ on $\bigoplus_{X} \mathbb{Z} / 2 \mathbb{Z}$ is amenable. This includes $X=\mathbb{Z}, \mathbb{Z}^{2}$ or more generally a metric space $(X, d)$ with bounded geometry that embeds coarsely in $\mathbb{Z}^{2}$.

- If $X$ contains a Lipschitz and injective image of the infinite binary tree, then the action of $\bigoplus_{X} \mathbb{Z} / 2 \mathbb{Z} \rtimes W(X)$ on $\bigoplus_{X} \mathbb{Z} / 2 \mathbb{Z}$ is not amenable.

The sufficient condition in terms of the random walk uses [10] and is a necessary and sufficient condition for a stronger condition to hold, see Remark 2.3. We also take the opportunity in Remark 2.4 to present an alternative proof, due to Narutaka Ozawa, of [10, Theorem 1.2].

By Remark 3.5, Question 1.2 has a negative answer for many Cayley graphs of groups with exponential growth. By [14, Theorem 3.24], the first criterion applies to a finitely generated group $X=\Gamma$ if and only if $\Gamma$ is virtually $\{0\}, \mathbb{Z}, \mathbb{Z}^{2}$. The case when $X=\mathbb{Z}^{d}, d \geq 3$ remains an intriguing open question.

By [13] a positive answer to Question [1.2 would follow from the weak amenability of $\bigoplus_{X} \mathbb{Z} / 2 \mathbb{Z} \rtimes W(X)$. We could not follow this approach, but this led us to wonder whether $W(X)$ can contain property $(\mathrm{T})$ subgroups.

As one may expect there is a strong relation between group structure of $W(X)$ and metric space structure of $X$. We show that if $X$ is of uniform subexponential growth, then $W(X)$ does not contain infinite property $(T)$ subgroups, see Theorem 4.1. On the other hand, an example of R. Tessera, see Theorem 4.3 shows that there exists a solvable group $\Gamma$ such that $W(\Gamma)$ contains $S L_{3}(\mathbb{Z})$.

The paper is organized as follows. In Section 2 we study the notion of transience for metric spaces with bounded geometry and prove the first half of Theorem 1.4. In Section 3 we prove the second half of Theorem 1.4 (Proposition 3.4), and in a last section we study when $W(X)$ contains property $(T)$ groups. 


\section{ACKNOWLEDGEMENTS}

We thank R. Tessera for interesting conversations and for allowing us to include Theorem 4.3. We also thank Y. de Cornulier for useful remarks on the presentation of Section 3. We are grateful to R. Grigorchuk for providing us with numerous comments and suggestions that improved the paper a lot. In a preliminary version of this paper our results were not stated in terms of random walks. U. Bader, B. Hua and A. Valette suggested a connection with recurrence of random walks and B. Hua pointed out [14, Theorem 3.24] to us. We thank them, and especially A. Valette, for this suggestion and for many other valuable comments. Finally we thank Narutaka Ozawa for his Remark 2.4.

\section{RECURRENT RANDOM WALKS AND AMENABILITY}

Here we prove the following fact on recurrent random walks.

Proposition 2.1. Let $(X, d)$ be a metric space with bounded geometry and $x_{0} \in X$. Then $(X, d)$ is recurrent if and only if for every finitely supported symmetric probability measure $\mu$ on $W(X)$, the random walk on $X$ starting from $x_{0}$ and jumping from $x$ to $g \cdot x$ according to the measure $\mu$ is recurrent.

Before we prove Proposition 2.1, we state some properties of transience for metric spaces.

Proposition 2.2. Let $(X, d)$ be a metric space with bounded geometry and $x_{0} \in X$. Let $R>0$ such that the random walk starting at $x_{0}$ and jumping from a point $x$ uniformly to $B(x, R)$ is transient. Then for every $R^{\prime}>R$ the random walk starting at $x_{0}$ and jumping from a point $x$ uniformly to $B(x, R)$ is also transient.

The notion of transience given by Definition 1.3 is independent of $x_{0}$.

In the case $(X, d)$ is a connected graph with bounded geometry, the transience in the sense of Definition 1.3 is equivalent to the transience of the usual random walk on the graph.

Proof. Consider $(V, E)$ the connected component of $x_{0}$ in the graph structure on $X$ where there is an edge between two points of $X$ at distance at most $R^{\prime}$. Then the random walk starting at $x_{0}$ and jumping from $x$ uniformly to $B(x, R)$ is a reversible random walk on $(V, E)$ with constant conductance and bounded range, so that by [14, Theorem 3.2] its transience implies the transience of the simple random walk on $(V, E)$. This proves the first point.

Let $x_{0}, x_{1} \in X$. If there is $R$ such that the random walk starting at $x_{0}$ and jumping from a point $x$ uniformly to $B(x, R)$ is transient, by the first point we can assume that $R>d\left(x, x_{0}\right)$, so that the same random walk starting at $x_{1}$ is also transient. This proves the second point.

Assume that $(X, E)$ is a connected graph with bounded geometry, take $x_{0} \in X$ and $R \geq 1$. Let $\left(X, E^{\prime}\right)$ be the graph structure on $X$ in which there is an edge between two points of $X$ at distance at most $R$. The formal identity between $(X, d)$ and $\left(X, d^{\prime}\right)$ is a bilipschitz bijection, so that by [14, Theorem 3.10] the random walk on $(X, E)$ is transient if and only if the random walk on $\left(X, E^{\prime}\right)$ is transient.

Proof of Proposition 2.1. Assume that $(X, d)$ is recurrent. Take $\mu$ as in the Proposition. Remember the notation (11) and pick $R>\max _{g \in \operatorname{supp}(\mu)}|g|_{w}$. Since $(X, d)$ is recurrent, the random walk starting at $x_{0}$ and jumping from a point $x$ uniformly to $B(x, R)$ is recurrent. By [14, Theorem 3.2] the random walk starting at $x_{0}$ and jumping from a point $x$ to $g \cdot x$ uniformly according to $\mu$ is therefore also recurrent.

Reciprocally, assume that $(X, d)$ is transient, and take $R>0$ as in the definition. We will construct a finite symmetric subset $S$ of $W(X)$ such for that every pair of points $x, y \in X$ at 
distance less than $R$ there is $g \in S$ such that $g x=y$. By [14, Theorem 3.2] this will imply the transience of the simple random walk on the connected component of $x_{0}$ in the graph structure on $X$ in which there is an edge between $x$ and $g x$ for every $x \in X, g \in S$. In other words if $\mu$ is the uniform probability measure on $S$, the random walk on $X$ starting from $x_{0}$ and jumping from $x$ to $g \cdot x$ according to $\mu$ is transient. Here is the construction of $S$. Define a graph structure on $X$ by putting an edge between $x$ and $x^{\prime}$ if $d\left(x, x^{\prime}\right) \leq R$. We obtain a (not necessarily connected) graph $(X, E)$ with bounded geometry on which the random walk starting from $x_{0}$ is transient. Denote by $d_{E}$ the associated graph distance. Take a finite collection $\left(X_{i}\right)_{i \leq l}$ of subsets of $X$ such that $\cup_{i} X_{i}=X$ and $d_{E}(x, y) \geq 3$ for all $x, y \in X_{i}$ and all $i$. Take $k \in \mathbb{N}$, and for every $x \in X$ take a sequence $y_{1}(x), \ldots, y_{k}(x)$ that covers all neighbours of $x$ in $(X, E)$. The existence of such collection $\left(X_{i}\right)$ and such $k$ follows from the bounded geometry assumption. Then for every $i \leq l$ and every $j \leq k$, consider the element $s_{i, j}$ of $W(X)$ that permutes $x$ and $y_{j}(x)$ for every $x \in X_{i}$ and acts as the identity on the rest of $X$. Then $S=\left\{s_{i, j}, i \leq l, j \leq k\right\}$ works. Indeed by construction for every neighbours $\left(x, x^{\prime}\right) \in(X, E)$ there is at least one (in fact two) element of $S$ that permutes $x$ and $x^{\prime}$.

Proof of the first part of Theorem 1.4. Assume that $(X, d)$ is recurrent. Let $G$ be a finitely generated subgroup of $W(X)$. By Proposition [2.1] and [10, Theorem 1.2], the action of $\bigoplus_{G x_{0}} \mathbb{Z} / 2 \mathbb{Z} \rtimes G$ on $\bigoplus_{G x_{0}} \mathbb{Z} / 2 \mathbb{Z}$ is amenable. This implies that the action of $\bigoplus_{X} \mathbb{Z} / 2 \mathbb{Z} \rtimes G$ on $\bigoplus_{X} \mathbb{Z} / 2 \mathbb{Z}$ is amenable : if $m$ is a $\bigoplus_{G x_{0}} \mathbb{Z} / 2 \mathbb{Z} \rtimes G$-invariant mean on $\ell_{\infty}\left(\bigoplus_{G x_{0}} \mathbb{Z} / 2 \mathbb{Z}\right)$, then $f \in \ell_{\infty}\left(\bigoplus_{X} \mathbb{Z} / 2 \mathbb{Z}\right) \mapsto m\left(f \bigoplus_{G x_{0}} \mathbb{Z} / 2 \mathbb{Z}\right)$ is a $\bigoplus_{X} \mathbb{Z} / 2 \mathbb{Z} \rtimes G$-invariant mean. This proves that the action of every finitely subgroup of $\bigoplus_{X} \mathbb{Z} / 2 \mathbb{Z} \rtimes W(X)$ on $\bigoplus_{X} \mathbb{Z} / 2 \mathbb{Z}$ is amenable, and concludes the proof.

Remark 2.3. As is well-known, the action of a group on a set $Y$ is amenable if and only if there is a net $f_{\alpha}$ of unit vectors in $\ell_{2}(Y)$ such that $\lim _{\alpha}\left\|g \cdot f_{\alpha}-f_{\alpha}\right\|=1$ for all $g \in G$. In the special case of $G=\bigoplus_{X} \mathbb{Z} / 2 \mathbb{Z} \rtimes W(X)$ acting on $Y=\bigoplus_{X} \mathbb{Z} / 2 \mathbb{Z}$, Proposition 2.1] and [10, Theorem 1.2] show that the recurrence of $(X, d)$ is equivalent to the existence of such a net $f_{\alpha}$ with the additional property that $f_{\alpha} \in \ell^{2}\left(\bigoplus_{X} \mathbb{Z} / 2 \mathbb{Z}\right)$ is of the form $f_{\alpha}(\omega)=\prod_{x \in X} f_{\alpha, x}\left(\omega_{x}\right)$ for functions $f_{\alpha, x}: \mathbb{Z} / 2 \mathbb{Z} \rightarrow \mathbb{C}$.

Remark 2.4. By the theory of electrical networks (see [10] for details), the Schreier graphs of a transitive action of a finitely generated group $G$ on $X$ carry a recurrent random walk if and only there exists a sequence of finitely supported function $a_{n}: X \rightarrow[0,1]$ that satisfy :

(1) $a_{n}\left(x_{0}\right)=1$

(2) $\lim _{n}\left\|g \cdot a_{n}-a_{n}\right\|_{\ell_{2}(X)}<\varepsilon$ for every $g \in G$.

It was proved in [10] (and used above) that this implies a positive answer to Question 1.1. We record here a slightly different proof, due to Narutaka Ozawa (personal communication). Let $\mathcal{P}_{f}(X)$ denote the set of all finite subsets of $X$, that we identify with $\oplus_{X} \mathbb{Z} / 2 \mathbb{Z}$. For $a_{n}$ as above, let $\xi_{n}(B)=\prod_{x \in B} a_{n}(x)$ for $B \in \mathcal{P}_{f}(X)$ and $\xi_{n}(\emptyset)=1$. Then $\xi_{n} \in \ell^{2}\left(\mathcal{P}_{f}(X)\right)$ is $\left\{x_{0}\right\}$-invariant and for $g \in G$

$$
\left\langle g \xi_{n}, \xi_{n}\right\rangle=\sum_{B \in \mathcal{P}_{f}(X)} \prod_{x \in B} a_{n}(x) a_{n}(g x)=\prod_{x \in X}\left(1+a_{n}(x) a_{n}(g x)\right)
$$


by distributivity. In particular for the identity element,

$$
\left\langle\xi_{n}, \xi_{n}\right\rangle=\prod_{x \in X}\left(1+a_{n}(x)^{2}\right)=\prod_{x \in X}\left(1+a_{n}(g x)^{2}\right)=\prod_{x \in X} \sqrt{1+a_{n}(x)^{2}} \sqrt{1+a_{n}(g x)^{2}}
$$

by reordering the terms. Therefore

$$
\begin{aligned}
& \log \frac{\left\langle\xi_{n}, \xi_{n}\right\rangle}{\left\langle g \xi_{n}, \xi_{n}\right\rangle}=\log \prod_{x \in X} \frac{\sqrt{1+a_{n}(x)^{2}} \sqrt{1+a_{n}(g x)^{2}}}{1+a_{n}(x) a_{n}(g x)} \\
& \quad \leq \sum_{x \in X} \frac{\left(a_{n}(x)-a_{n}(g x)\right)^{2}}{2\left(1+a_{n}(x) a_{n}(g x)\right)^{2}} \leq \frac{1}{2}\left\|a_{n}-g \cdot a_{n}\right\|_{\ell^{2}(X)}^{2}
\end{aligned}
$$

which goes to zero as $n \rightarrow \infty$. The first inequality is the basic inequality $\ln (\sqrt{1+A}) \leq \frac{1}{2} A$ for

$$
A=\frac{\left(1+a_{n}(x)^{2}\right)\left(1+a_{n}(g x)^{2}\right)}{\left(1+a_{n}(x) a_{n}(g x)\right)^{2}}-1=\frac{\left(a_{n}(x)-a_{n}(g x)\right)^{2}}{\left(1+a_{n}(x) a_{n}(g x)\right)^{2}} .
$$

Any weak-* cluster point in $\ell_{\infty}\left(\mathcal{P}_{f}(X)\right)^{*}$ of the sequence $\left|\xi_{n}\right|^{2} /\left\|\xi_{n}\right\|^{2}$ will therefore be a $G \ltimes$ $\mathcal{P}_{f}(X)$-invariant mean. This construction of $\xi_{n}$ should be compared to the one in [10], which was defined (through Fourier transform) as $\xi_{n}(B)=\prod_{x \in B} \sin \left(\frac{\pi}{4} a_{n}(x)\right) \times \prod_{x \notin B} \cos \left(\frac{\pi}{4} a_{n}(x)\right)$.

\section{Negative answer to the Question 1.2}

Let $G$ be a group acting on $X$. We start by recording the following result. The second assertion follows from results proved later, but is not used in the rest of the paper.

Proposition 3.1. If the action of $\bigoplus_{X} \mathbb{Z} / 2 \mathbb{Z} \rtimes G$ on $\bigoplus_{X} \mathbb{Z} / 2 \mathbb{Z}$ is amenable, then so is the action of $G$ on $X$. The converse is not true.

Proof. Assume that the action of $\bigoplus_{X} \mathbb{Z} / 2 \mathbb{Z} \rtimes G$ on $\bigoplus_{X} \mathbb{Z} / 2 \mathbb{Z}$ amenable. By (ii) implies (iv) in [9, Lemma 3.1], the set $\mathcal{P}_{f}^{*}$ of non-empty finite subsets of $X$ carries a $G$-invariant mean $m$. Consider the unital positive $G$-equivariant map $T: \ell_{\infty}(X) \rightarrow \ell_{\infty}\left(\mathcal{P}_{f}^{*}\right)$ given by $T f(A)$ is the average of $f$ on $A$, for all $A$ nonempty finite subset of $X$. The composition $m \circ T$ is a $G$-invariant mean on $X$.

To see that the converse is not true, take for $X$ the Cayley graph of a finitely generated amenable group $\Gamma$ that contains an infinite binary tree (see Remark 3.5 for the existence of such group). By Theorem 1.4 , the action of $\bigoplus_{X} \mathbb{Z} / 2 \mathbb{Z} \rtimes W(X)$ on $\bigoplus_{X} \mathbb{Z} / 2 \mathbb{Z}$ is not amenable. On the other hand the action of $W(X)$ on $X$ is amenable; more precisely any $\Gamma$-invariant mean $m$ on $X$ is also $W(X)$-invariant. Indeed, for any $g \in W(X)$ there is a finite partition $A_{1}, \ldots, A_{n}$ of $X$ and elements $\gamma_{1}, \ldots, \gamma_{n}$ such that $g$ acts as the translation by $\gamma_{k}$ on $A_{k}$. Then for every $f \in \ell_{\infty}(X)$, using that $\left(\gamma_{i}\left(A_{i}\right)\right)_{i=1}^{n}$ forms a partition of $X$ we get

$$
m(g \cdot f)=\sum_{i} m\left(\gamma_{i} \cdot\left(f 1_{\gamma_{i}\left(A_{i}\right)}\right)=\sum_{i} m\left(f 1_{A_{i}}\right)=m(f) .\right.
$$

When $X$ is the Cayley graph of a finitely generated group, the first assertion in Proposition 3.1 implies

Lemma 3.2. Let $\Gamma$ be a finitely generated group. If there exists a $\bigoplus_{\Gamma} \mathbb{Z} / 2 \mathbb{Z} \rtimes W(\Gamma)$-invariant mean on $\bigoplus_{\Gamma} \mathbb{Z} / 2 \mathbb{Z}$ then $\Gamma$ is amenable. 
We can also give a negative answer to Question 1.2 for some amenable groups. One ingredient for this is the following monotonicity property.

Lemma 3.3. Let $i: X \rightarrow Y$ an injective map such that $\sup _{d\left(x, x^{\prime}\right) \leq R} d\left(i(x), i\left(x^{\prime}\right)\right)<\infty$ for every $R>0$. If Question 1.2 has a positive answer for $Y$, then is also has positive answer for $X$.

Proof. In this proof we denote by $\mathcal{P}_{f}(X)$ the set of all finite subsets of $X$, which carries a natural action of $W(X)$. It follows from the equivalence of (ii) and (iv) in [9, Lemma 3.1] that Question 1.2 has a positive answer if and only if there is a $W(X)$-invariant mean on $\mathcal{P}_{f}(X)$ giving full weight to the subsets containing any given element of $X$.

The map $i$ allows to define an embedding $W(X) \subset W(Y)$ by defining, for $g \in W(X)$, $g \cdot i(x)=i(g \cdot x)$ and $g \cdot y=y$ if $y \notin i(X)$.

Assume that Question 1.2 has a positive answer for $Y$, and take $x_{0} \in X$. By [9, Lemma 3.1] there is a mean $m$ on $\mathcal{P}_{f}(Y)$ that is $W(Y)$-invariant and that gives full weight to the collection of sets containing $i\left(x_{0}\right)$. Then the push-forward mean on $\mathcal{P}_{f}(X)$ (given by $\varphi \in$ $\ell_{\infty}\left(\mathcal{P}_{f}(X)\right) \mapsto m\left(A \mapsto \varphi\left(i^{-1}(A)\right)\right)$ is $W(X)$-invariant and gives full weight to the collection of sets containing $x_{0}$. By [9, Lemma 3.1] again, Question[1.2 has a positive answer for $X$.

Lemma 3.3 and Proposition 3.1 imply that for $\bigoplus_{X} \mathbb{Z} / 2 \mathbb{Z}$ to act amenably on $\bigoplus_{X} \mathbb{Z} / 2 \mathbb{Z}$ it is necessary that $W\left(X^{\prime}\right)$ act amenably on $X^{\prime}$ for all $X^{\prime} \subset X$. In particular the following Proposition establishes the second half of Theorem 1.4.

Proposition 3.4. Let $(X, d)$ be a metric space with bounded geometry with an injective and Lipschitz map from the infinite binary tree $T$ to $X$. Then there is no $\bigoplus_{X} \mathbb{Z} / 2 \mathbb{Z} \rtimes W(X)$ invariant mean on $\bigoplus_{X} \mathbb{Z} / 2 \mathbb{Z}$.

Proof. There is a Lipschitz injective map from the free group with two generators in $T$, and hence in $X$ if $X$ contains an injective and Lipschitz image of $T$. The Proposition therefore follows from Lemma 3.2 and Lemma 3.3 ,

Remark 3.5. The class of groups for which this proposition applies, i.e. for which there is a Cayley graph that contains a copy of the infinite binary tree as a subgraph, contains in particular all non-amenable groups ([2, Theorem 1.5]), as well as all elementary amenable groups with exponential growth (by [4] such groups contain a free subsemigroup). In [6], R. Grigorchuk, disproving a conjecture of Rosenblatt, proved that the lamplighter group $Z_{2} \imath G$ contains an infinite binary tree, here $G$ is Grigorchuk's 2-group of intermediate growth. We do not know whether all groups with exponential growth contain such a tree.

\section{Property (T) Subgroups}

It is an interesting question to extract properties of the group $W(X)$ using the properties of the underlying metric space. Below we prove that $W(X)$ cannot contain property $(T)$ groups when $X$ is of subexponential growth. Alain Valette (personal communication) pointed out to us that a very similar observation (atttributed to Kazhdan) was made by Gromov in [8] Remark 0.5.F: a discrete property (T) group $G$ cannot contain a subgroup $G^{\prime}$ such that $G / G^{\prime}$ has subexponential growth unless $G / G^{\prime}$ is finite.

Theorem 4.1. Let $X$ be a metric space with uniform subexponential growth :

$$
\lim _{n} \frac{1}{n} \log \sup _{x \in X}|B(x, n)|=0 \text {. }
$$


Then $W(X)$ does not contain an infinite countable property $(T)$ group.

Proof. Assume $G<W(X)$ is a finitely generated property $(T)$ group, with finite symmetric generating set $S$. We will prove that $G$ is finite. To do so we prove that the $G$-orbits on $X$ are finite, with a uniform bound. Assume that $1 \in S$. If $m=\max \left\{|g|_{w}: g \in S\right\}$, then $S^{n} x \subset B(x, m n)$ for every $x \in X$, so that by assumption, the growth of $S^{n} x$ is subexponential (uniformly in $x \in X$ ). The classical expanding properties for actions of $(T)$ groups will imply that the orbit of $x$ is finite (uniformly in $x$ ).

Indeed, by $(T)$, there exists $\varepsilon>0$ such that for every unitary action of $G$ on a Hilbert space $H$ without invariant vectors, the inequality $\sum_{g \in S}\|g \cdot \xi-\xi\|^{2} \geq \varepsilon\|\xi\|^{2}$ holds for every $\xi \in H$. As a consequence, for every transitive action of $G$ on a set $Y$, we have $\sum_{g \in S}|g F \Delta F| \geq \varepsilon / 2|F|$ for every finite subset $F$ of $Y$ satisfying $2|F| \leq|Y|$ (take $H=\ell_{2}(Y)$ if $Y$ is infinite, and $H=$ the subspace of $\ell_{2}(Y)$ orthogonal to the vector with all coordinates equal otherwise, and apply the preceding equality with $\xi=\chi_{F}-|F| /|Y \backslash F| \chi_{Y \backslash F}$. Here $\chi_{F}$ is the indicator function of $F$, and $|F| /|Y \backslash F|$ is by convention 0 if $Y$ is infinite). By induction, we therefore have that for $x \in Y$ and $n \in \mathbb{N},\left|S^{n} x\right| \geq(1+\varepsilon / 4)^{n}$ unless $|Y| \leq 2(1+\varepsilon / 4)^{n}$. Applying it to the orbit of some $x \in X$, we get

$$
\left|S^{n} x\right|<(1+\varepsilon / 4)^{n} \Longrightarrow\left|\operatorname{Orb}_{G}(x)\right|<2(1+\varepsilon / 4)^{n} .
$$

Hence, subexponential growth gives an $n \in \mathbb{N}$ such that $\left|\operatorname{Orb}_{G}(x)\right|<2(1+\varepsilon / 4)^{n}$ for every $x \in X$. QED.

To construct spaces such that $W(X)$ contains property $(\mathrm{T})$ groups, we first remark that the groups $W(X)$ behave well with respect to coarse embeddings. A map $q:\left(X, d_{X}\right) \rightarrow\left(Y, d_{Y}\right)$ between metric spaces is a coarse embedding if there exists nondecreasing functions $\varphi_{+}, \varphi_{-}$: $\left[0, \infty\left[\rightarrow \mathbb{R}\right.\right.$ such that $\lim _{t \rightarrow \infty} \varphi_{-}(t)=\infty$ and

$$
\varphi_{-}\left(d_{X}\left(x, x^{\prime}\right)\right) \leq d_{Y}\left(q(x), q\left(x^{\prime}\right)\right) \leq \varphi_{+}\left(d_{X}\left(x, x^{\prime}\right)\right)
$$

for every $x, x^{\prime} \in X$.

Lemma 4.2. Let $q:\left(X, d_{X}\right) \rightarrow\left(Y, d_{Y}\right)$ be a map such that there is an increasing function $\varphi_{+}: \mathbb{R}^{+} \rightarrow \mathbb{R}^{+}$such that $d_{Y}(q x, q y) \leq \varphi_{+}\left(d_{X}(x, y)\right)$, and such that the preimage $q^{-1}(y)$ of every $y \in Y$ has cardinality less than some constant $K$ (e.g. $q$ is a coarse embedding and $X$ has bounded geometry). Let $F$ be a finite metric space of cardinality $K$. Then $W(X)$ is isomorphic to a subgroup of $W(Y \times F)$.

Proof. In this statement $Y \times F$ is equipped with the distance $d\left((y, f),\left(y^{\prime}, f^{\prime}\right)\right)=d_{Y}\left(y, y^{\prime}\right)+$ $d_{F}\left(f, f^{\prime}\right)$. Since $F$ is bigger than $q^{-1}(y)$ for all $y$, there is a map $f: X \rightarrow F$ such that the map $\widetilde{q}: x \in X \mapsto(q(x), f(x)) \in Y \times F$ is injective. We can therefore define an action of $W(X)$ on $Y \times F$ by setting $g(\widetilde{q}(x))=\widetilde{q}(g x)$ and $g(y, f)=(y, f)$ if $(y, f) \notin \widetilde{q}(X)$. The assumption on $\varphi_{+}$guarantees that this action is by wobblings, ie that it defines an embedding of $W(X)$ in $W(Y \times F)$.

In a contrast to Theorem 4.1 we have the following result by Romain Tessera. With his kind permission we include a proof.

Theorem 4.3. There is a solvable group $\Gamma$ such that $W(\Gamma)$ contains the property $(T)$ group $S L(3, \mathbb{Z})$. 
INVARIANT MEANS FOR THE WOBBLING GROUP

Proof. The proof uses the notion of asymptotic dimension (see [1]). By [1, Corollary 94], $S L(3, \mathbb{Z})$ has finite asymptotic dimension. By [1, Theorem 44] this implies that $S L(3, \mathbb{Z})$ embeds coarsely into a finite product of binary trees. Take $\Gamma_{0}$ a solvable group with a free semigroup. In particular it coarsely contains a binary tree, so $S L(3, \mathbb{Z})$ embeds coarsely in $\Gamma_{0}^{n}$ for some $n$. By Lemma 4.2, there is a finite group $F$ such that $W(S L(3, \mathbb{Z}))$ embeds as a subgroup in $W\left(F \times \Gamma_{0}^{n}\right)$. But $W(S L(3, \mathbb{Z}))$ contains $S L(3, \mathbb{Z})$ (action by translation).

Remark 4.4. The proof actually shows that for every group $\Lambda$ with finite asymtotic dimension, there is an integer $n$ such that $\Lambda$ is isomorphic to a subgroup of $W\left(\Gamma^{n}\right)$ whenever there is a Cayley graph of $\Gamma$ that contains an infinite binary tree as a subgraph. By Remark 3.5 this includes lots of groups $\Gamma$ with exponential growth. In some sense this says that the assumptions of Theorem 4.1 are not so restrictive.

\section{REFERENCES}

[1] G. Bell, A. Dranishnikov, Asymptotic Dimension, Topology Appl. 12 (2008) 1265-1296.

[2] I. Benjamini And O. Schramm, Every graph with a positive Cheeger constant contains a tree with a positive Cheeger constant, Geometric and Functional Analysis 7 (1997), 3, 403-419

[3] T. Ceccherini-Silberstein, R. Grigorchuk, P. De la Harpe, Amenability and paradoxical decompositions for pseudogroups and discrete metric spaces, Proc. Steklov Inst. Math. 1999, no. 1 (224), 57-97.

[4] C. Chou, Elementary amenable groups, Illinois J. Math. 24 (1980), 3, 396-407.

[5] W. A. Deuber, M. Simonovits, V. T. Sós, A note on paradoxical metric spaces, Studia Sci. Math. Hungar. 30 (1995), no. 1-2, 17-23.

[6] R. Grigorchuk, Superamenability and the occurrence problem of free semigroups, (Russian) Funktsional. Anal. i Prilozhen. 21 (1987), no. 1, 74-75.

[7] R. Grigorchuk, K. Medynets, On algebraic properties of topological full groups, Mat. Sb. 205 (2014), no. $6,87-108$.

[8] M. Gromov, Asymptotic invariants of infinite groups, Geometric group theory, Vol. 2, London Math. Soc. Lecture Note Ser. 182 (1993).

[9] K. Juschenko, N. Monod, Cantor systems, piecewise translations and simple amenable groups Ann. of Math. (2) 178 (2013), no. 2, 775-787.

[10] K. Juschenko, V. Nekrashevych, M. DE LA Salle, Extensions of amenable groups by recurrent groupoids, arXiv:1305.2637

[11] M. LaczKovich, Equidecomposability and discrepancy; a solution of Tarski's circle-squaring problem, J. Reine Angew. Math. 404 (1990) 77-117.

[12] H. MatuI, Some remarks on topological full groups of Cantor minimal systems, Internat. J. Math. 17 (2006), no. 2, 231-251.

[13] N. Ozawa, Examples of groups that are not weakly amenable, Kyoto J. Math. 52 (2012), no. 2, 333-344.

[14] W. Woess, Random walks on infinite graphs and groups, Cambridge Tracts in Mathematics (2000).

Kate Juschenko: Northwestern University \& CNRS, Université Lyon 1

Mikael de la Salle: CNRS, ENS de Lyon. 